\title{
Bildungspolitik und der Sozialinvestitionsstaat
}

\author{
Marius R. Busemeyer und Julian L. Garritzmann
}

\section{Zusammenfassung}

Spätestens seit dem Wandel der westlichen Demokratien von Industrie- zu Wissensgesellschaften sind Bildung und Bildungspolitik zentrale Themen der vergleichenden Sozialstaatsforschung geworden. Dieses Kapitel bietet einen Überblick über die politikwissenschaftliche, historisch-vergleichende Literatur zu Bildungspolitik und diskutiert das komplexe Zusammenspiel von Bildungs- und Sozialpolitik. Im zweiten Teil des Kapitels stellen wir Sozialinvestitionspolitik als ein neues Paradigma der Sozialpolitikforschung vor und diskutieren dessen politökonomische Dynamik und Effekte.

\section{$1 \quad$ Einleitung}

Investitionen in Bildung bzw. „Humankapital“ gelten in Zeiten voranschreitender Globalisierung und vor dem Hintergrund des strukturellen Wandels der Industriezu wissensbasierten Dienstleistungsökonomien als wichtiges und vielversprechendes Instrument, um wirtschaftliche Entwicklung mit dem sozialpolitischen Anspruch der sozialen Gerechtigkeit und Inklusion zu verbinden (Boix 1998). Aber inwiefern kann und sollte Bildungspolitik tatsächlich als funktionales Äquivalent anderer Sozialpolitiken dienen? Und wie variabel stellt sich das Verhältnis von Bildungs- und Sozialpolitik in unterschiedlichen Zeitperioden und Länderkontexten dar? Diesen Fragen möchten wir im vorliegenden Kapitel nachgehen und dabei kurz den Stand der Forschung darstellen. Dabei zeigen wir auch, dass und warum Bildungspolitik erst recht kürzlich zum Analyseobjekt der vergleichenden Sozialpolitikforschung geworden und somit ein junger, wenn auch rasant wachsender Teilbereich ist. 
In den folgenden Abschnitten gehen wir zunächst auf das Verhältnis von Bildungsund Sozialpolitik aus einer historisch-vergleichenden Perspektive ein. Hierbei wird auch die Frage angesprochen, inwiefern Bildungspolitik - in Analogie zu anderen Bereichen des Sozialstaates - zur Reduzierung sozioökonomischer Ungleichheit beitragen kann. Der zweite große Teil dieses Kapitels schließt unmittelbar an die aktuelle Debatte um das Modell des "Sozialinvestitionsstaates" an (Bonoli 2013; Esping-Andersen 2002; Hemerijck 2013, 2017; Morel et al. 2012), das von vielen Politikern und Sozialpolitikforschern als das neue Wohlfahrtsstaatsparadigma angesehen wird. Diese Debatte wird und wurde in den letzten Jahren auf unterschiedlichen Ebenen (national/international; wissenschaftlich/politisch) geführt und hat die Frage nach dem Verhältnis zwischen Bildungs- und Sozialpolitik auf neue Art und mit neuer Dringlichkeit gestellt.

\section{Historisch-vergleichende Perspektiven auf das Verhältnis von Bildungs- und Sozialpolitik}

\subsection{Das komplexe Verhältnis von Bildungs- und Sozialpolitik}

Viele Beiträge, die das Verhältnis von Bildungs- und Sozialpolitik diskutieren, erwähnen zu Beginn Harold Wilenskys (1975, S. 3) einflussreiche These: „Education is special." Wilensky bezog sich auf die Tatsache, dass Bildungspolitik im Unterschied zu anderen Sozialpolitiken weniger direkt - und häufig auch weniger umfassend zu materieller Umverteilung beiträgt. Aus diesem Grund - so Wilensky - sollte Bildung nicht unbedingt als integraler Bestandteil von Wohlfahrtsstaats-Regimen betrachtet werden, sondern als ein eigenständiger Bereich, der bestenfalls lose mit dem Rest des Sozialstaates verknüpft ist. Ein gewichtiges Gegenargument gegen die „Abkopplungs-These" von Wilensky basiert auf der Annahme, dass Bildungsinstitutionen - wie andere sozialpolitische Institutionen - für die Verteilung von Lebens- und Bildungschancen, für Einkommen auf dem Arbeitsmarkt und damit letztlich auch für sozioökonomische Ungleichheit relevant sind. Auch wenn die Zusammenhänge komplexer und die Umverteilungseffekte weniger offensichtlich sind, kann und sollte Bildungspolitik letztlich auch aus einer redistributiven Perspektive analysierbar sein.

Diese Perspektive dominiert in politisch-ökonomischen Analysen der historischen und gegenwärtigen Entwicklungsdynamik von Bildungspolitik, die parteipolitische Auseinandersetzungen um die Gestaltung von Bildung in den Mittelpunkt rücken (Ansell 2010; Boix 1998; Busemeyer 2015; Garritzmann 2016; Schmidt 2007). So könnte Wilensky (und anderen) entgegengehalten werden, dass der Wohlfahrtstaat neben der (Um-)Verteilung von Ressourcen auch andere Funktionen erfüllt, beispielweise die Bereitstellung und Förderung von Humankapital, was zu einer Verbesserung der wirtschaftlichen Leistungsfähigkeit und der Beschäftigungspotentiale in einem Land führen kann. Diese Argumentationslinie findet sich in Beiträgen der 
Abbildung 1 Zusammenhang zwischen öffentlichen Bildungs- und Sozialausgaben (2014).

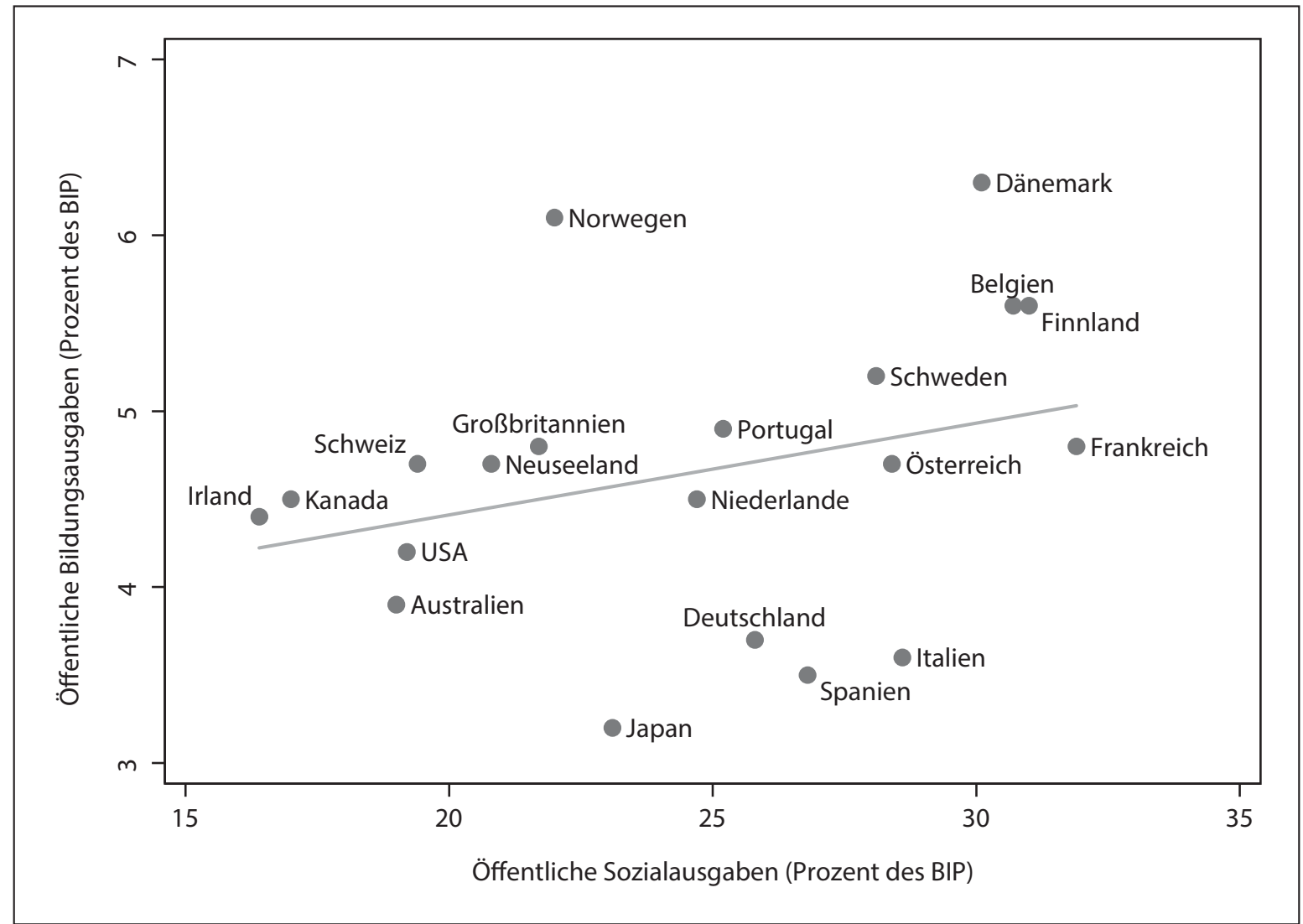

Quellen: Öffentliche Bildungsausgaben: OECD (2017), Education at a Glance 2017: OECD Indicators, OECD Publishing, Paris, S. 189; Öffentliche Sozialausgaben: OECD (2016), OECD Factbook 2015-2016: Economic, Environmental and Social Statistics, OECD Publishing, Paris, S. 191.

„Varieties of Capitalism“-Schule (Hall und Soskice 2001; Iversen und Soskice 2001; Estévez-Abe et al. 2001; Cusack et al. 2006) sowie bei der - besonders bei Ökonomen prominenten - Theorie des „endogenen Wachstums“ (Barro 2001). Beide Perspektiven werden weiter unten ausführlicher diskutiert. Zunächst soll jedoch ein kurzer Blick auf einige empirische Zusammenhänge zur Motivation der Diskussion geworfen werden.

Abbildung 1 zeigt den Zusammenhang zwischen öffentlichen Bildungs- und Sozialausgaben, jeweils gemessen als Anteil am Bruttoinlandsprodukt für das Jahr 2014. Die Abbildung belegt zunächst einen tendenziell positiven Zusammenhang zwischen öffentlichen Bildungs- und Sozialausgaben, was darauf hindeutet, dass beide Bereiche auf ähnliche politische und historische Ursprünge zurückgehen könnten. Gleichzeitig zeigen sich unterschiedliche Profile, was die relative Bedeutung angeht: Dänemark und Norwegen geben beispielsweise deutlich mehr für Bildung aus, als man auf Grundlage des Niveaus der Sozialausgaben erwarten würde. Spanien und Italien, aber auch Deutschland und Japan, geben umgekehrt vergleichsweise viel für Sozialpolitik aus und wenig für Bildung. In vielen Ländern, zum Beispiel in Frankreich, Schweden, 
den Niederlanden und Österreich, gehen hohe Sozialausgaben mit überdurchschnittlich hohen Bildungsausgaben einher, in anderen Ländern (insbesondere Irland, Kanada und den USA) ist es aber umgekehrt.

Wenn man über den relativ engen Betrachtungsrahmen der Staatsfinanzen hinausgeht, gibt es weitere auffällige Parallelen in der institutionellen Ausgestaltung von Bildungs- und Wohlfahrtsstaats-Regimen (Busemeyer und Nikolai 2010; Busemeyer 2015; Mosher 2015; Willemse und de Beer 2012). Dieser Befund lässt Zweifel aufkommen, ob Wilenskys These („Education is special“) tatsächlich zutrifft. Wenn Bildungspolitik politisch und institutionell losgelöst von anderen Bereichen des Sozialstaates stattfinden würde, dürfte es auf der Makro-Ebene keinen Zusammenhang zwischen den beiden Bereichen geben. Der internationale Vergleich zeigt allerdings, dass der Typus universalistischer Wohlfahrtsstaaten skandinavischer Prägung einhergeht mit integrativ ausgestalteten Bildungsinstitutionen, d.h. nach Gesamtschulprinzip organisierten Sekundarschulen, großzügiger öffentlicher Subventionierung von Bildungsteilnahme und weithin offenen Zugängen zu höheren Sektoren des Bildungssystems. In „konservativ“-kontinentaleuropäischen Wohlfahrtsstaaten finden sich hingegen häufig stark gegliederte Sekundarschulsysteme, die Kinder im frühen Alter auf verschiedene Bildungswege verteilen, aber auch durch starke Berufsbildung und vergleichsweise gering ausgebaute Hochschulsektoren gekennzeichnet sind. In den „liberalen“ (vornehmlich angelsächsischen) Wohlfahrtsstaaten schließlich spielen private Bildungsinstitutionen eine wesentlich größere Rolle. Das liberale Erbe manifestiert sich in einem dem skandinavischen Modell nicht unähnlichen Primarund Sekundarschulsystem mit Gesamtschul-Fokus, sowie in einem ausdifferenzierten und weithin offenen Hochschulsektor, der allerdings auch eine hohe institutionelle Stratifizierung aufweist (Allmendinger 1989). Außerdem spielen im liberalen Modell private Bildungseinrichtungen eine viel größere Rolle.

Zweitens kommen auch Zweifel hinsichtlich der Argumentation Arnold Heidenheimers (1981) auf, der - in einer weiteren zentralen These der Bildungspolitikforschung - Bildungs- und Sozialpolitik als funktional äquivalente Entwicklungspfade unterschiedlicher Wohlfahrtstaaten-Regime betrachtete. Heidenheimer (1981) konzentrierte sich hierbei auf den Vergleich der historischen Entwicklungspfade der USA mit europäischen Ländern (Deutschland, Schweden, Vereinigtes Königreich) und stellte fest, dass die USA im Vergleich zu den europäischen Ländern sehr viel früher das öffentliche Bildungswesen ausgebaut und dadurch den Zugang zu Bildung geöffnet hatten. Im Unterschied dazu betrieben europäische Länder, insbesondere Deutschland, zunächst eine Expansion der Sozialversicherungssysteme (Rente, Gesundheit, Arbeit), bevor nach dem Zweiten Weltkrieg die Bildungsexpansion auch in Europa ihre volle Kraft entfalten konnte. In diesem Sinne beschreibt Heidenheimer zwei unterschiedliche Pfade, die sich zwar im Zeitpunkt und der Sequenzierung wichtiger Ereignisse unterscheiden, im Kern aber zu einem ähnlichen Ergebnis führen, nämlich einem umfassend ausgebauten Wohlfahrtsstaat. Bildungs- und Sozialpolitik sind in Heidenheimers Perspektive somit Substitute: Manche Länder 
(insbesondere die angelsächsischen) investieren in Bildung, aber weniger in Sozialleistungen; andere (insbesondere die konservativen) investieren in Sozial-, aber weniger in Bildungspolitik.

In Heidenheimers Argumentation schwingt der Optimismus der Modernisierungstheoretiker der 196oer Jahre mit, und er selbst hat wohl die Wahrscheinlichkeit der Einführung einer universalen öffentlichen Krankenversicherung in den USA - wie viele andere - überschätzt. Für die Diskussion in diesem Kapitel bleibt jedenfalls festzuhalten, dass heute beträchtliche Unterschiede zwischen OECD-Ländern hinsichtlich des Verhältnisses von Bildungs- und Sozialpolitik bestehen. Diese deuten eher darauf hin, dass sich beide Bereiche komplementär zueinander verhalten („Bildung und Sozialpolitik") und weniger als funktionale Äquivalente, die wie in einem System „kommunizierender Röhren“ einander substituieren können („Bildung statt Sozialpolitik“). Die „Komplementaritäts-These“ geht letztlich zurück auf T. H. Marshall, der in seinem Sozialrechts-Katalog das Recht auf Bildung („the right of the adult citizen to have been educated“, Marshall [1964: 81-82]) gleichberechtigt neben soziale und

Abbildung 2 Zusammenhang zwischen privaten Bildungsausgaben und öffentlichen Sozialausgaben (2014).

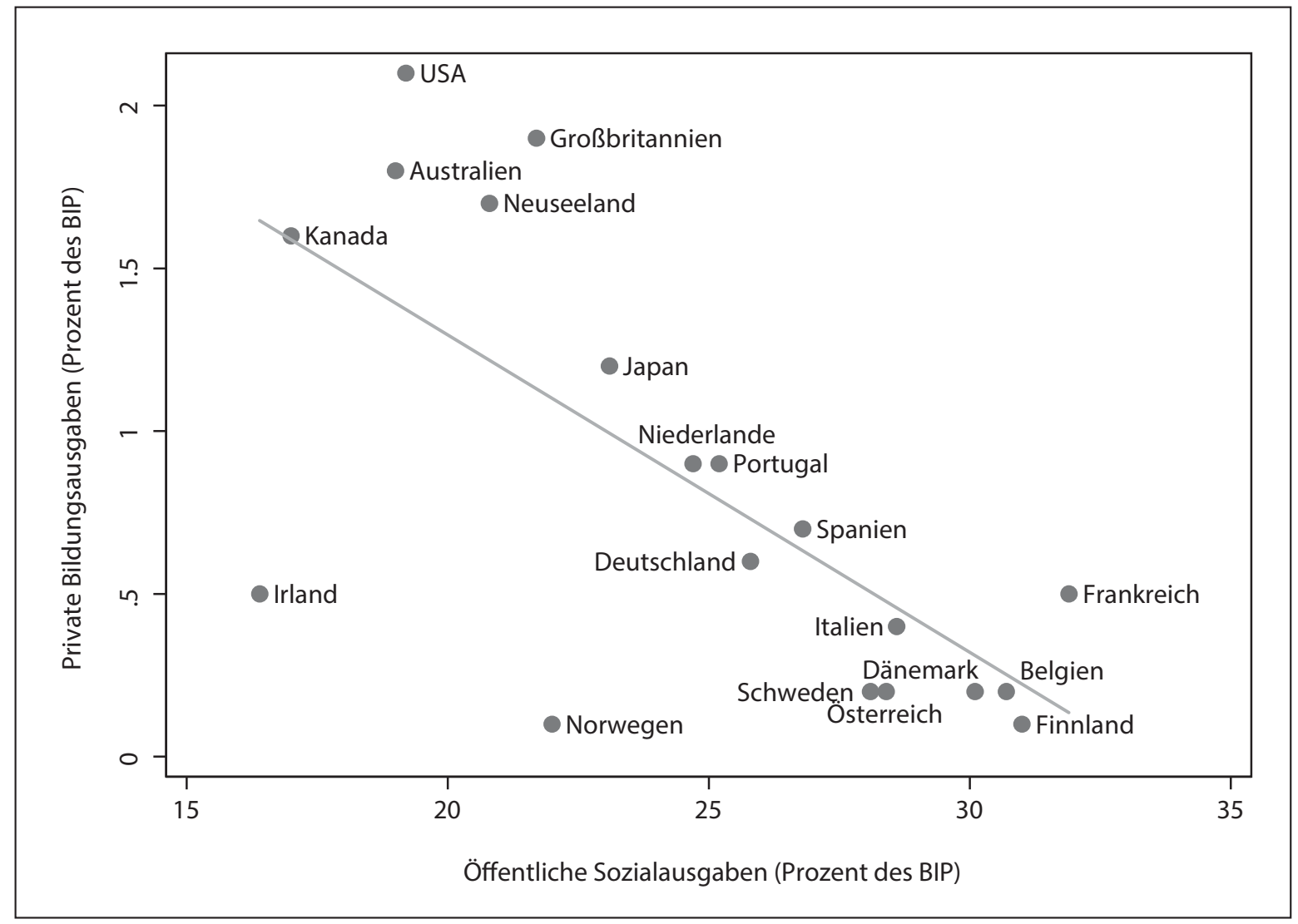

Quellen: Private Bildungsausgaben: OECD (2017), Education at a Glance 2017: OECD Indicators, OECD Publishing, Paris, S. 189; Öffentliche Sozialausgaben: OECD (2016), OECD Factbook 2015-2016: Economic, Environmental and Social Statistics, OECD Publishing, Paris, S. 191. 
politische Beteiligungsrechte gestellt und so das Bild eines umfassenden Wohlfahrtsstaates geprägt hat.

Heidenheimers „Substitutions-Perspektive“ bekommt allerdings Unterstützung, wenn man die Analyseperspektive auf den Bereich der privaten Ausgaben erweitert. Abbildung 2 zeigt einen negativen Zusammenhang zwischen der Höhe der privaten Bildungsausgaben (in Prozent des BIP) und den öffentlichen Sozialausgaben (ebenfalls für das Jahr 2014). Weitere Analysen zeigen, dass Länder mit unterdurchschnittlichen öffentlichen Bildungsausgaben diese teilweise durch höhere private Bildungsausgaben kompensieren können. Da es sich bei privaten Bildungsausgaben vor allem um Studiengebühren und nur zu geringen Teilen um Beiträge anderer privater Akteure (wie Stiftungen oder Unternehmen) handelt, gilt dies allerdings nur für einige Länder, in denen das Hochschulsystem entsprechend institutionell ausgestattet ist (beispielsweise die USA, Kanada, Großbritannien, Japan, Australien oder Neuseeland). Somit findet Heidenheimers These der funktionalen Äquivalenz eine gewisse Unterstützung in dem Sinne, dass die Macht der Bildungsexpansion, die sich heutzutage vor allem auf den Ausbau des Zugangs zum Hochschulsektor fokussiert, über viele Länder hinweg und über längere Zeiträume betrachtet zu höheren öffentlichen oder privaten Bildungsinvestitionen geführt hat. Beträchtliche Länderunterschiede bleiben allerdings bestehen hinsichtlich der Art und Weise, wie das Verhältnis zwischen privater und öffentlicher Finanzierung und Trägerschaft ausgestaltet ist.

\subsection{Erklärungsansätze: Machtressourcen, Parteipolitik und "Varieties of Capitalism"}

Die vergleichende Policy-Analyse verfügt mittlerweile über einen ausdifferenten Katalog an Theorien, die dabei helfen, Variationen im policy output im Quer- und Längsschnittvergleich zu erklären (siehe die Beiträge im ersten Teil dieses Handbuchs). In der Analyse der Bildungspolitik spielen maßgeblich zwei Theorie-Stränge eine Rolle, auf die wir uns im Folgenden konzentrieren wollen: die Machtressourcen- und Parteiendifferenztheorie einerseits und der "Varieties of Capitalism"-Ansatz andererseits.

Beiträge in der Tradition der Machtressourcen- und Parteiendifferenztheorie (vgl. auch die Beiträge von Ebbinghaus und Zohlnhöfer i. d. B.) erklären Unterschiede im policy output mit Unterschieden in der Verteilung von Machtressourcen zwischen den kollektiv organisierten Vertretern der Interessen von Kapital (Arbeitgeberverbände) und Arbeit (Gewerkschaften). Parteiendifferenztheoretische Perspektiven fokussieren weniger auf außerparlamentarische Machtressourcen, sondern stärker auf das Handeln politischer Parteien im Rahmen ihrer Regierungstätigkeit. In diesem Sinne können sich parteiendifferenztheoretische Ansätze auch leichter gegenüber neuen Ansätzen der Parteien- und Wahlforschung öffnen (Häusermann et al. 2013): Sie betonen die Strategiefähigkeit parteipolitischer Eliten in Reaktion auf sich ändernde Parteienwettbewerbsdynamiken und betrachten Parteien anders als die klas- 
sische Machtressourcentheorie nicht als verlängerte Transmissionsriemen außerparlamentarischer Interessen in Regierung und Parlament, wie es noch Douglas Hibbs (1977) in der ursprünglichen Version der Parteiendifferenztheorie getan hatte.

Aus machtressourcen- und parteiendifferenztheoretischer Perspektive lassen sich die Parallelen in der institutionellen Ausgestaltung von Bildungssystemen und Wohlfahrtsregimen recht einfach dadurch erklären, dass die Ideologien und Interessen derjenigen politischen Akteure, die in historischen Phasen kritischer Weichenstellungen an der Macht waren, ihre Vorstellungen gleichermaßen in der Sozial- und der Bildungspolitik durchsetzen konnten. Das skandinavische Modell des universalistischen Wohlfahrtsstaates ist wohl das eindrücklichste Beispiel für diesen Zusammenhang. Vom Anspruch her umfasste das "nordische“ Modell des universalistischen Wohlfahrtsstaates nicht nur die Sozialpolitik im engeren Sinne, sondern auch den Bildungssektor (Antikainen 2006). Im kontinentaleuropäischen, konservativ-korporatistischen Modell mögen diese Zusammenhänge mehr indirekt und weniger explizit, aber dennoch wirkmächtig gewesen sein. Der starke Einfluss christdemokratischer Ideologie in diesem Regime (van Kersbergen 1995) ging einher mit einer gewissen Zurückhaltung hinsichtlich staatlicher Interventionspolitik und dem Respekt vor politischer und institutioneller Eigenständigkeit der Bildungspolitik. Dies spiegelt sich in einer Präferenz für gegliederte Sekundarschulsysteme und einer eigenständigen Berufsbildung wider, zumindest in der Länderfamilie der „kollektiven Ausbildungssysteme" (Busemeyer und Trampusch 2012).

Durch den Aufstieg der "Varieties of Capitalism"-Schule (VoC) (siehe Paster i. d. B.) sind machtressourcen- und parteiendifferenztheoretische Perspektiven auf grundsätzliche Weise herausgefordert worden. Das Politikfeld Bildung spielte eine zentrale Rolle in diesen Debatten, die so auch dazu beigetragen haben, die politikwissenschaftliche Bildungsforschung von einer peripheren Stellung in der vergleichenden Policy-Analyse stärker in den Mainstream zu bringen (Busemeyer und Trampusch 2011; Gift und Wibbels 2014). Bildung - bzw. „skills“ - spielen im VoC-Ansatz eine, wenn nicht die, zentrale Rolle, so dass Bildungssysteme und -politik zu einem Kernthema der Vergleichenden Politischen Ökonomie geworden sind. Einen ersten wichtigen Beitrag zu dieser Diskussion lieferten die Untersuchungen von Kathleen Thelen (2004) zu den historischen Ursprüngen von Ausbildungssystemen in unterschiedlichen „Spielarten“ des Kapitalismus. Thelen arbeitete heraus, dass politische Auseinandersetzungen um die Organisation und Finanzierung von Ausbildung historisch sehr unterschiedliche Verläufe nahmen. Der entscheidende Unterschied zwischen „liberalen Marktwirtschaften" und "koordinierten Marktwirtschaften“ lag allerdings nicht darin, dass sich in ersteren die Machtbalance klar in Richtung Kapital und in letzteren in Richtung Arbeit verlagert hätte, wie die Machtressourcentheorie annahm. Vielmehr betonte Thelen, dass sich koordinierte Marktwirtschaften dadurch auszeichnen, dass Ausbildungspolitik auf einem klassenübergreifenden Kompromiss zwischen Arbeitgebern und Gewerkschaften beruht und auf diese Weise langfristig stabile Muster von Kooperation und Koordination produziert werden. 
Der Grundgedanke der klassenübergreifenden Kooperation bzw. Koordination zwischen Arbeitgebern und Arbeitnehmern als zentrales Merkmal von koordinierten Marktwirtschaften findet sich auch in anderen Beiträgen der VoC-Schule (Iversen und Soskice 2001; Estévez-Abe et al. 2001; Hall und Soskice 2001). Aus der VoC-Perspektive war der zentrale Beitrag der Sozialpolitik, individuelle Investitionen in $\mathrm{Hu}-$ mankapital abzusichern und damit erst zu ermöglichen (Iversen und Soskice 2001; Estévez-Abe et al. 2001; Cusack et al. 2006). Das Hauptargument dabei ist, dass Arbeitnehmern nur dann finanzielle und zeitliche Ressourcen in für Firmen relevante Fähigkeiten (skills) investieren, wenn sie sozialpolitisch gegen Arbeitsmarkt-Risiken abgesichert sind. Sozialpolitik wird in dieser Perspektive also zum "Steigbügelhalter" der Bildungspolitik.

Ohne hier ausführlich auf die Gegenkritik der Machtressourcentheorie an der VoC-Schule eingehen zu können, sei lediglich darauf verwiesen, dass einer der Hauptkritikpunkte darin besteht, dass die grobe Unterscheidung zwischen liberalen (z. B. USA, Großbritannien) und koordinierten Marktwirtschaften (z. B. Deutschland, Schweden, Dänemark) wenig hilfreich ist, um die signifikanten Unterschiede in der Ausgestaltung der Bildungssysteme innerhalb der Gruppe der koordinierten Marktwirtschaften erklären zu können. So wird beispielsweise der Unterschied zwischen skandinavischen und kontinentaleuropäischen Systemen ausgeblendet (Busemeyer 2015; Mosher 2015; Streeck 2012; Thelen 2014). Insofern liegt eine Erweiterung der VoC-Perspektive um machtressourcen- und parteidifferenztheoretische Perspektiven nahe (Busemeyer 2015; Garritzmann 2016; Iversen und Stephens 2008), nicht zuletzt, weil der VoC-Ansatz oft ahistorisch und funktionalistisch argumentiert.

\subsection{Bildungs- und sozioökonomische Ungleichheit}

Wie stellt sich das Verhältnis zwischen Bildungs- und Sozialpolitik auf der Ebene der sozioökonomischen Outcomes dar? Abbildung 3 liefert hier zunächst einen ernüchternden Befund. Auf der vertikalen Achse ist das Ausmaß der Lohn-Ungleichheit (Quotient zwischen Einkommen auf dem 9. Dezil zu Einkommen auf dem 1. Dezil) abgetragen; auf der horizontalen Achse das Ausmaß von Bildungsungleichheit, operationalisiert durch einen Indikator, der die Stärke des Zusammenhangs zwischen familiärem sozioökonomischen Hintergrund und Bildungsleistungen im Rahmen der PISA-Studie misst. Je höher dieser Wert, desto höher das Ausmaß der Bildungsungleichheit. Abbildung 3 zeigt, dass es bestenfalls nur einen sehr schwachen $\mathrm{Zu}$ sammenhang zwischen Bildungs- und Lohnungleichheit gibt. Wenn, dann wäre der Zusammenhang außerdem tendenziell negativ (höhere Bildungsungleichheit geht einher mit geringerer Lohnungleichheit). Tiefergehende Analysen, die unterschiedliche Operationalisierungen und Indikatoren für Bildungs- und sozioökonomische Ungleichheit verwenden, bestätigen diesen „Nicht-Befund“ (Busemeyer 2015; Solga 2014). 
Abbildung 3 Zusammenhang zwischen Bildungs- und sozioökonomischer Ungleichheit (2012).

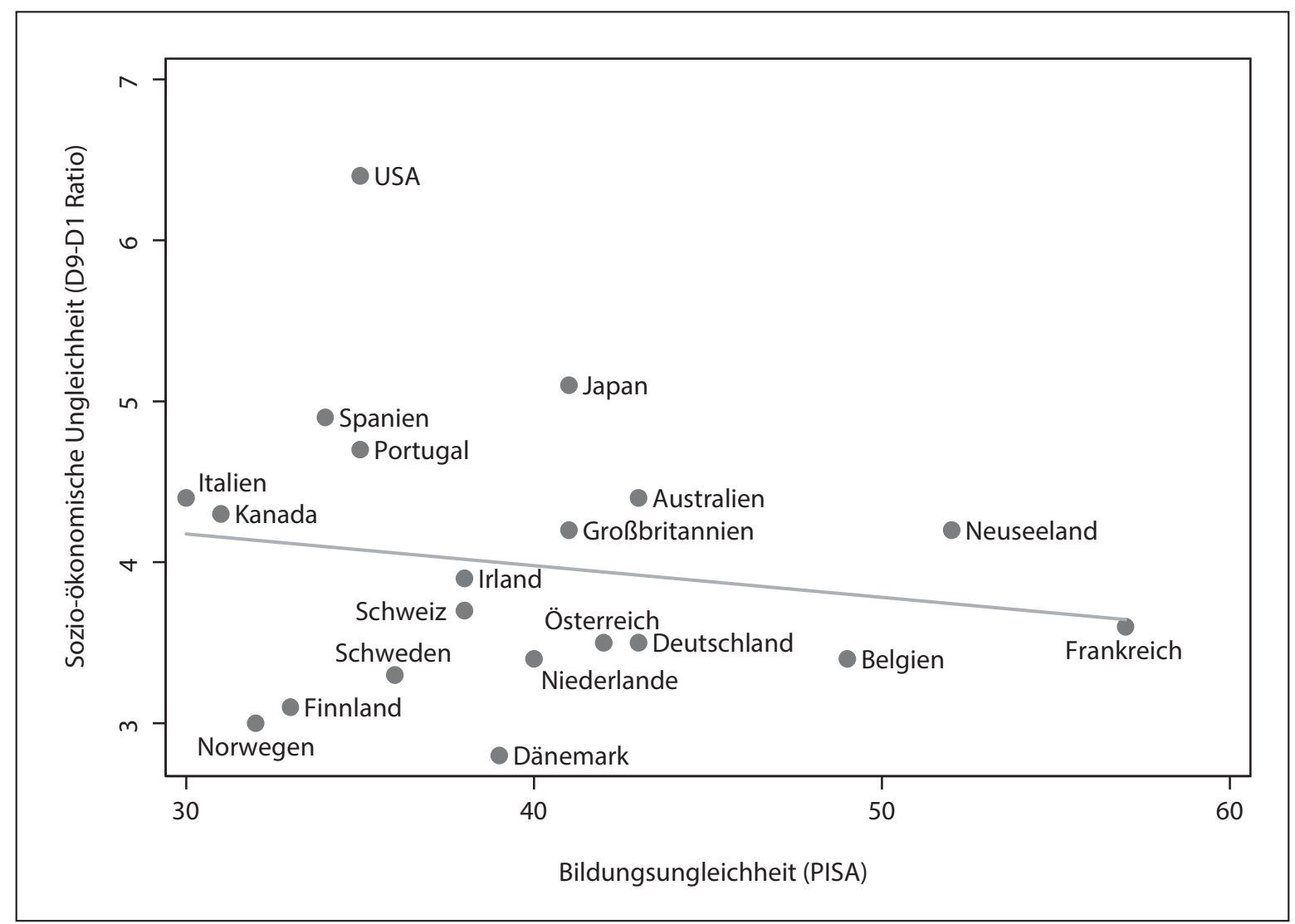

Quelle: OECD Statistics Database, OECD (2013), PISA 2012 Results: Excellence Through Equity: Giving Every Student the Chance to Succeed (Volume II), PISA, OECD Publishing, S. 106.

Auf den ersten Blick scheint dies eindeutig ein Punktsieg für Wilensky zu sein: Bildungspolitik scheint - im Ländervergleich - ein weniger effektives Instrument der Umverteilung als andere Sozialpolitiken zu sein, zumal der Wohlfahrtsstaat und sein Steuer- und Transfersystem einen seit langem anerkannten Beitrag zur Verminderung sozioökonomischer Ungleichheit leisten (Bradley et al. 2003). Aus einer theoretischen Perspektive kann dieser „Nicht-Befund“ jedoch durch die Tatsache erklärt werden, dass Bildungsinvestitionen nicht nur öffentlichen, sondern auch privaten Nutzen erzeugen: Zwar kann der Ausbau des Zugangs zum Hochschulsektor langfristig den Wohlstand eines Landes fördern, indem qualifizierte Fachkräfte ausgebildet werden. Er führt aber auf der individuellen Ebene auch dazu, dass diejenigen, die in den Genuss dieser Bildungsleistungen kommen, hohe Bildungs- „Renditen“ einfahren können, so dass sich die Lohnungleichheit vergrößern könnte.

Tiefergehende Analysen des Zusammenhangs zwischen Bildungs- und sozioökonomischer Ungleichheit haben gezeigt, dass der scheinbare „Nicht-Zusammenhang“ auf der hochaggregierten Makro-Ebene differenzierter ausfällt, wenn man sich spezifische Aspekte der institutionellen und finanziellen Ausgestaltung von Bildungs- 
systemen anschaut (vgl. zum Folgenden Busemeyer 2015, Kap. 4). Zwei Faktoren spielen hier eine besondere Rolle. Der erste Faktor betrifft das Verhältnis zwischen privater und öffentlicher Finanzierung der Bildung (Wolf und Zohlnhöfer 2009): Ein hoher Anteil privater Bildungsausgaben geht einher mit einem höheren Ausmaß sozioökonomischer Ungleichheit (Busemeyer 2015, S. 194). Dies gilt insbesondere für den Hochschulsektor, in dem private Bildungsausgaben eine besonders große Rolle spielen und Ungleichheiten verstärken (Garritzmann 2016). Der zweite Faktor, der im Zusammenhang mit Bildungs- und sozioökonomischer Ungleichheit relevant ist, nimmt weniger die Finanzierung, sondern stärker die institutionelle Struktur des Bildungssystems in den Blick: Hier zeigt die empirische Analyse, dass das Ausmaß sozioökonomischer Ungleichheit in Ländern geringer ist, die über ein ausgebautes und gut institutionalisiertes Berufsbildungssystem verfügen (Busemeyer 2015, S. 191; vgl. auch Estévez-Abe et al. 2001). Ein gut ausgebautes Berufsbildungssystem eröffnet Jugendlichen aus bildungsfernen Schichten auch Zugänge zu Aus- und Weiterbildung sowie sich anschließender gut bezahlter Beschäftigung, die es in Ländern mit einer starken Fokussierung auf die hochschulische, akademische Bildung im post-sekundären Sektor nicht gibt. Außerdem tragen qualitativ hochwertige (duale) Ausbildungssysteme signifikant zu geringer Jugendarbeitslosigkeit bei (Breen 2005).

\section{Der Aufstieg des „Sozialinvestitionsstaats“ als Reformmodell}

Im zweiten Teil dieses Kapitels wenden wir uns nun einer aktuellen und fortlaufenden Debatte in der Wohlfahrtsstaatsforschung und -politik zu: dem Aufstieg des „Sozialinvestitionsstaats", ein Modell, das in der Sozialstaatsforschung und der Politik vielfach als das neue (zukünftig) führende sozialpolitische Paradigma betrachtet wird (Esping-Andersen 2002; Jenson 2010; Morel et al. 2012; Hemerijck 2013, 2017). Wie im Folgenden gezeigt wird, stellt Bildung und Bildungspolitik ein zentrales Charakteristikum des Sozialinvestitionsstaats dar, weswegen wir es hier in diesem Kapitel diskutieren.

\subsection{Zur Definition von „Sozialinvestitionen“}

Die Kernidee der „Sozialinvestitionen“ und des „Sozialinvestitionsstaat“-Modells ist „to aim at ,preparing' rather than ,reparing“" (Morel et al. 2012, S. 1; ähnlich: EU Commission 2013): Während klassische Sozialpolitik auf ex post-Entschädigung von Einkommens- und Arbeitsplatzverlusten zielt, beispielsweise durch Arbeitslosengeld, will vorbeugende Sozialpolitik die Bürger ex ante mit Fähigkeiten ausstatten, so dass sie überhaupt erst keine Verluste erfahren oder zumindest wieder schnell auf den Arbeitsmarkt zurückkehren können. Sozialinvestitionspolitik stellt dabei Bildung und (arbeitsmarktrelevante) Fähigkeiten (skills) ins Zentrum sozialstaatlicher Aktivität 
und versucht, die Sozialpolitik auf dieses Ziel auszurichten. Anders als kompensierende Sozialpolitik, die auf hohe De-Kommodifizierung abzielt, also die Ermöglichung eines soliden Lebensstandards auch ohne Arbeitsmarktbeteiligung (Esping-Andersen 1990; Obinger und Petersen und Manow i. d. B.), ist das Ziel des Sozialinvestitionsstaates somit eher die (Re-)Kommodifizierung, also die Ermöglichung der Arbeitsmarktteilnahme aller. Sozialpolitik wird als „produktiver Faktor“ verstanden, der nicht per se gegen Marktkräfte gerichtet ist („politics against markets“, Esping-Andersen 1985), sondern diese nutzt, um soziale Inklusion und wirtschaftliche Leistungsfähigkeit zu fördern („politics for markets“, Iversen und Soskice 2015). Hier spielt auch die Reduktion von Ungleichheiten zwischen Geschlechtern eine zentrale Rolle, denn Sozialinvestitionspolitik zielt insbesondere auf die Arbeitsmarktintegration von Frauen bzw. auf eine geschlechterneutrale Familienpolitik.

Typische Sozialinvestitionspolitiken sind somit (1) Politiken, die auf frühkindliche Bildung und Erziehung abzielen (Morgan 2006), (2) schulische und hochschulische Bildungspolitik (Busemeyer 2015; Garritzmann 2016), (3) aktive, qualifikationsorientierte und unterstützende Arbeitsmarktpolitik (Bonoli 2010; Vlandas 2013; Weishaupt i. d. B.) sowie (4) Familienpolitik, die eine Vereinbarkeit von Familie und Beruf sowie Geschlechtergerechtigkeit anstrebt (Häusermann 2018; Bothfeld und Rouault 2015). Dies ist aber keine abschließende Liste, denn durch die breiten gesellschaftlichen und ökonomischen Ziele des Ansatzes können viele weitere policies ebenso den Charakter von Sozialinvestitionen annehmen. Ein zu breites Verständnis von Sozialinvestitionspolitiken birgt allerdings die Gefahr des „concept stretching“ und der definitorischen Beliebigkeit. So gibt es beispielsweise Diskussionen um die Frage, ob conditional cash transfers, die in Lateinamerika und vielen Schwellenländern zu einem der wichtigsten sozialpolitischen Werkzeuge geworden sind, als Sozialinvestitionspolitiken verstanden werden sollten, da sie einerseits dieselben Ziele wie Sozialinvestitionspolitik haben, andererseits aber in der Form von monetären Sozialtransfers und nicht als soziale Dienstleistungen ausgehändigt werden.

Was aber ist Sozialinvestitionspolitik, wenn sie so umfassend verstanden wird und sozialinvestitionspolitische Elemente in fast jedem Politikbereich vorhanden sind (Kvist 2015)? Ist das Sozialinvestitionskonzept nur , alter Wein in neuen Flaschen (Nolan 2013)? In der Tat scheint „Sozialinvestition“ ein immer beliebterer Slogan in der Forschung wie in der Politik geworden zu sein, insbesondere auf der EU-Ebene sowie in supranationalen Organisationen. Es besteht daher die Gefahr, dass der Begriff durch eine immer breitere Verwendung zu einem allumfassenden und damit nichtssagenden Etikett für wünschenswerte Politiken und Ergebnisse wird und damit an analytischer Schärfe verliert. Julian Garritzmann, Silja Häusermann und Bruno Palier argumentieren daher, dass eine klare Definition essentiell ist und definieren Sozialinvestitionspolitiken als „policies that create, mobilize, or preserve human skills“ (Garritzmann et al. 2017, S. 37). Anhand dieser Definitionen kann für jede konkrete Politik entschieden werden, ob sie als Sozialinvestition verstanden werden kann oder nicht. Diese Definition zeigt auch, dass Bildung, aber auch die Beschäfti- 
gungsfähigkeit und die Verbindung zum Arbeitsmarkt im Konzept des Sozialinvestitionsstaats die zentrale Rolle spielen.

\subsection{Zwei Modelle des Sozialinvestitionsstaats}

Zur Vieldeutigkeit des Sozialinvestitionsstaats-Modells trägt bei, dass es zwei gleichermaßen populäre Verständnisse und Traditionen des Konzeptes gibt (Jenson 2010; Morel et al. 2012): Auf der einen Seite steht eine skandinavische Variante des Sozialinvestitionsmodells, in dem der Ausbau sozial-investiver Policies nicht als Substitut, sondern als komplementäre Ergänzung zu großzügig ausgestalteter, kompensierender Sozialpolitik angesehen wird. Vertreter dieser Variante (bspw. Esping-Andersen 2002; Hemerijck 2013, 2017) betonen, dass kompensierende und vorbeugende Sozialpolitik zusammenarbeiten müssen, um die ambitionierten sozialen und ökonomischen Ziele des Sozialinvestitionsstaates zu erreichen. Historisch lässt sich die skandinavische Variante des Sozialinvestitionsstaates in Schweden bis in die 1930er-Jahre zum Modell des ,productivist welfare states' zurückverfolgen (Morel et al. 2012, S. 5). ${ }^{1}$ Aus skandinavischer Perspektive ist der Sozialinvestitionsstaat demnach auch kein aktuelles „Reformmodell“, sondern vielmehr ein längst etabliertes Faktum. Eine Diskussion findet hier eher um den zukünftigen Bestand des universalistischen Wohlfahrtsmodells statt, der durch jüngere Entwicklungen - wie die inzwischen durchaus langjährige Regierungstätigkeit von bürgerlich-liberalen Koalitionen in einigen skandinavischen Ländern - herausgefordert wird (van Kersbergen und Kraft 2017; Bengtsson et al. 2017).

Auf der anderen Seite steht eine angelsächsische Variante des Sozialinvestitionsmodells, in dem Sozialinvestitionen eher als Substitut kompensierender Sozialpolitik statt als komplementäre Ergänzung wahrgenommen werden. Als intellektueller Vater dieser Variante kann der Soziologe Anthony Giddens (1998) verstanden werden, der einen „Third Way“, einen Mittelweg zwischen „linker“ und „rechter“ Politik propagierte und Sozialinvestitionen als „Kompromiss“ von expansiver, umverteilender, linker Sozialpolitik und fiskalkonservativer, subsidiärer, bürgerlich-liberaler Sozialpolitik vorschlug. Giddens' Ideen waren und sind nicht zuletzt deshalb so erfolgreich gewesen, weil er als Politikberater des damaligen Regierungschefs Tony Blair dessen Politik direkt beeinflussen konnte. Es geht hierbei also nicht um die Erweiterung des bestehenden, kompensatorischen Sozialstaates um eine sozial-investive Komponente, sondern um die Transformation des klassischen Sozialstaats in Richtung Sozialinvestitionen im engeren Sinne.

1 Mittlerweile wird der Begriff „productivist welfare state“ zur Charakterisierung der ostasiatischen Wohlfahrtsstaaten verwendet (Holliday 200o) um zu bezeichnen, wie in diesen Ländern die Sozialpolitik immer auf wirtschaftliche Politik und outcomes ausgerichtet ist. 
Die konzeptionellen Unterschiede zwischen den beiden Modellen konkretisieren sich am deutlichsten an ihrem unterschiedlichen Verständnis von "Aktivierung“ in der Arbeitsmarktpolitik (Bonoli 2010; Vlandas 2013; siehe auch Weishaupt i. d. B.). Während in der skandinavischen Variante „Aktivierung“ bedeutet, Arbeitslose oder prekär Beschäftigte mit Weiterbildungsmaßnahmen oder durch Unterstützung bei der Arbeitssuche auf einen neuen Arbeitsplatz vorzubereiten, beinhaltet das ThirdWay-Aktivierungsverständnis vor allem Maßnahmen, die Arbeitssuchende durch Unterstützung bei der Stellensuche sowie der Leistungskürzungen oder ähnliche Maßnahmen - sogenannte „negative reinforcements“ (Bonoli 2010) oder „workfare“Policies (King 1995) - zu einem schnelleren Arbeitsmarkteintritt zu bewegen suchen.

\subsection{Empirische Evidenz für die Verbreitung des Sozialinvestitionsmodells}

Unsere bisherige Vorstellung des Sozialinvestitions-Ansatzes fand vor allem auf der konzeptionellen und ideellen Ebene statt. Wie unterscheiden sich die OECD-Länder aber empirisch bezüglich des Verhältnisses zwischen vorbeugender und kompensierender Sozialpolitik? Zeigen sich ähnliche Muster wie in der klassischen SozialstaatsTypologie (siehe Manow i. d. B.) oder sind die „Welten des Wohlfahrtskapitalismus“ bei Sozialinvestitionen anders zusammengesetzt?

Wir können an dieser Stelle aus Platzgründen nur eine sehr grobe Skizze über die (wie immer vielschichtige) empirische Evidenz liefern und fokussieren auf öffentliche Staatsausgaben als Indikator für Staatstätigkeit. Vergleichen wir die Ausgabehöhe von Sozialinvestitionen und kompensierender Sozialpolitik, so zeigen sich sowohl interessante Gemeinsamkeiten als auch wichtige Unterschiede relativ zu den etablierten Typologien von Wohlfahrts-Regimen (siehe auch Bouget et al. 2015; Kuitto 2016; Nikolai 2012; Ronchi 2016). In Abbildung 4 zeigen wir auf der vertikalen Achse die Höhe der Sozialinvestitionen als Anteil am BIP; auf der horizontalen Achse haben wir die Ausgabenhöhe für kompensierende Sozialpolitik als Anteil am BIP abgetragen. Die Daten beziehen sich auf das Jahr 2010.

Abbildung 4 teilt die Länder in vier Gruppen ein, und zwar anhand des Kriteriums, ob sie über oder unter dem Länderdurchschnitt (dargestellt durch die schwarzen, durchgezogenen Linien) liegen. In der oberen rechten Ecke finden sich Länder, die vergleichsweise viel für Sozialinvestitionen sowie für kompensierende Sozialpolitik ausgeben. Hier finden wir neben den meisten skandinavischen Ländern auch Belgien, Frankreich und Österreich. Diese Länder ähneln am meisten dem skandinavischen Modell des Sozialinvestitionsstaats, in dem hohe Ausgaben für Sozialinvestitionen mit hohen Ausgaben für kompensierende Policies einhergehen. Länder in der oberen linken Ecke entsprechen eher dem Third-Way-Modell: Hier sind die Sozialinvestitionen ebenfalls vergleichsweise hoch, die für kompensierende Politik allerdings unterdurchschnittlich. Wie zu erwarten ist dies der Fall im Vereinigten Königreich, aber auch in Neuseeland, Irland, Luxemburg, Island und Norwegen. Die beiden zuletzt 
Abbildung 4 Öffentliche Ausgaben für Sozialinvestition und kompensierende Sozialpolitik, 2010.

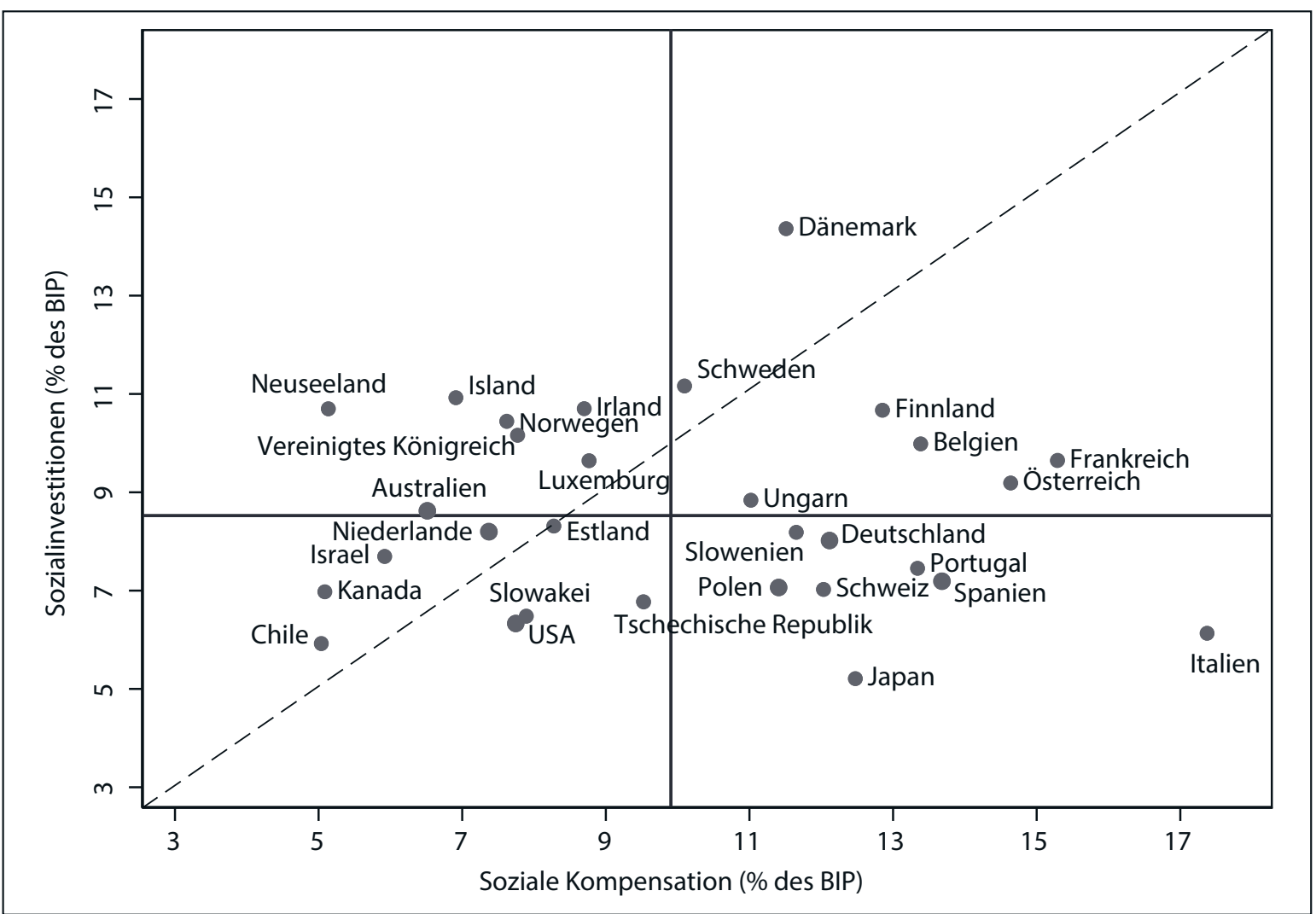

Quelle: Als relativ grobes, aber einschlägiges Maß - wir folgen hier Nikolai (2012) - beinhalten Sozialinvestitionen hier alle öffentlichen Ausgaben für Bildung, aktive Arbeitsmarktpolitik und Familien; kompensierende Sozialpolitik besteht aus Ausgaben für Renten und Arbeitslosenunterstützung. Die Bildungsausgabendaten stammen aus der Datenbank der Weltbank (The World Bank Data 2017 Online), alle anderen aus der Datenbank der OECD (OECD Stats 2017 Online).

genannten Beispiele zeigen somit, dass auch innerhalb der Gruppe der skandinavischen Staaten signifikante Unterschiede hinsichtlich der Schwerpunktsetzung auf die beiden Teilbereiche bestehen. Unten rechts in Abbildung 4 sind Länder mit der gegenteiligen Kombination: hohe Kompensationsausgaben und niedrige Sozialinvestitionen. Neben allen südeuropäischen Ländern finden sich hier Deutschland und die Schweiz sowie Japan und Slowenien. In der unteren linken Ecke schließlich sind Länder abgebildet, die auf beiden Dimensionen vergleichsweise geringe Staatsausgaben haben. Dies sind neben den USA und Kanada auch Chile, Israel, die Niederlande, sowie Tschechien und die Slowakei. Insgesamt zeigen sich somit zwar teilweise Überschneidungen mit den üblichen „Wohlfahrts-Regimen“ (Esping-Andersen 1990; Manow i. d. B.), aber auch zahlreiche Unterschiede. Norwegen, Island, Frankreich und Österreich werden beispielweise in Kategorien einsortiert, die nicht den regime-typischen Erwartungen entsprechen.

Um den Vergleich anzureichern haben wir in Abbildung 4 eine diagonal verlaufende gestrichelte Linie abgetragen. Länder, die über dieser Linie liegen, geben mehr 
für Sozialinvestitionen als für Kompensation aus, und Länder, die darunterliegen, relativ mehr für Kompensation und weniger für Sozialinvestitionen. Dieser Blickwinkel zeigt ein klareres Bild: Alle skandinavischen und alle angelsächsischen Länder priorisieren Sozialinvestitionen, während alle kontinental- und südeuropäischen Staaten (mit Ausnahme Luxemburgs) der Kompensation den Vorrang geben. Insbesondere Italien zeigt dabei einen enorm starken Fokus auf Kompensation und eine auffällige Vernachlässigung von Sozialinvestitionen (zum italienischen Fall siehe auch Kazepov und Ranci 2017; León und Pavolini 2014). Diese Analyseperspektive weist darüber hinaus auf einige Parallelen hin zum Vergleich der öffentlichen Bildungs- und Sozialausgaben (vgl. Abb. 1 oben).

\subsection{Die Politics des Sozialinvestitionsstaats}

Warum haben einige Länder weiter entwickelte Sozialinvestitionspolitiken als andere? Welche politischen, institutionellen oder sozioökonomischen Faktoren könnten diese Unterschiede erklären? Und inwiefern können die etablierten Theorien der Wohlfahrtsstaatenforschung sinnvoll angewendet werden? Eine zentrale These der bisherigen Forschung zur politischen Dimension des Sozialinvestitionsmodells besagt, dass sich die politische Dynamik der Sozialinvestitionspolitik grundlegend von derjenigen der klassischen Sozialpolitik unterscheidet. Allerdings wissen wir über die politics von Sozialinvestitionen insgesamt immer noch viel weniger als über die politische Dimension klassischer Sozialpolitik (Garritzmann et al. 2017 für einen detaillierten Literaturüberblick).

Worin liegen die zentralen Unterschiede in der politischen Dynamik? Erstens wird argumentiert, dass Sozialinvestitionspolitik vor allen Dingen in Reaktion auf das Aufkommen von „neuen soziale Risiken“ (Esping-Andersen 2002) entsteht. Im Gegensatz zu „alten sozialen Risiken“ aus der Zeit der Industrialisierung (beispielsweise arbeitsbedingte physische Gesundheitsrisiken) werden unter „neuen sozialen Risiken" die für das postindustrielle Zeitalter charakteristischen Gefährdungen verstanden, wie etwa die De-Standardisierung der Erwerbsarbeit (siehe dazu auch Eichhorst und Marx i. d. B.), sich verändernde Familienstrukturen, sowie der strukturelle Wandel von Industrie- zu Dienstleistungs- und Wissensgesellschaften (Powell und Snellman 2004), in denen ein zunehmender Fokus auf (sich ständig aktualisierenden) kognitiven Fähigkeiten liegt.

Neben diesen sozioökonomischen Faktoren spielen auch zahlreiche politische Einflüsse eine wichtige Rolle zur Erklärung von Unterschieden im Ausbau des Sozialinvestitionsstaates. Die politische Dynamik der Sozialinvestitionspolitik unterscheidet sich in dreierlei Hinsicht wesentlich von der Dynamik klassisch umverteilender Sozialpolitik. Erstens verläuft der politische Konflikt über Sozialinvestitionen entlang anderer politischer Konfliktlinien als in der klassischen kompensierenden Sozialpolitik. Während sich - im Sinne der Machtressourcentheorie (vgl. Ebbinghaus i. d. B.) - 
der Konflikt über Arbeitslosengeld-, Renten- oder Gesundheitspolitik in der Regel vor allem entlang der klassischen Links-Rechts- bzw. Kapital-Arbeit-Konfliktlinie äußert, gilt dies in viel geringerem Maße für Sozialinvestitionen. Häusermann (2012) zeigt beispielsweise, dass Sozialinvestitionspolitiken von einer klassenübergreifenden Koalition von neuen linken Parteien, liberalen Parteien und Arbeitgebern unterstützt werden, während traditionelle linke und konservative Parteien diesen Reformen kritisch gegenüberstehen können (vgl. Garritzmann et al. 2018 für eine detaillierte Analyse von Wählerpräferenzen). Der Konflikt um den Ausbau sozial-investiver Policies hat - zweitens - einen starken Bezug zum Generationen-Cleavage, da der Ausbau von Sozialinvestitionen tendenziell jüngeren Bevölkerungsgruppen hilft, während der Rückbau klassisch-kompensatorischer Politiken ältere Bevölkerungsgruppen negativ betreffen könnte. Zum Dritten aktivieren Sozialinvestitionspolitiken einen kulturellen Wertekonflikt, da viele sozialinvestive Policies implizit mit einem (post-)modernen Geschlechter- und Familienverständnis einhergehen und damit traditionellere Schichten herausfordern.

Als Hauptunterstützer von Sozialinvestitionspolitik werden verschiedene gesellschaftliche Gruppen genannt: (1) die Mittelschicht, die wenig von klassisch-redistributiver Sozialpolitik profitiert, aber durchaus von sozial-investiven Policies, vor allen Dingen, wenn sie die Vereinbarkeit von Familie und Beruf erleichtern; (2) Frauen, die sowohl als Arbeitnehmerinnen wie auch als Mütter, im traditionellen MaleBreadwinner-Modell hauptverantwortlich für die Hausarbeit sind, doppelt von Sozialinvestitionen profitieren; und (3) junge Wähler, für die diese Themen besondere Relevanz haben. Repräsentiert werden diese Gruppen in der Regel vor allem von neuen linken Parteien, von (sozial-)liberalen Parteien, die Sozialinvestitionen gutheißen, insofern sie häufig auf Marktmechanismen basieren, aber auch Chancengleichheit ermöglichen, und von grünen Parteien. Außerdem sind supranationale Organisationen - beispielswese UNICEF in Lateinamerika und die OECD in Europa - als wichtige Akteure zu nennen, die das Sozialinvestitionsstaats-Modell als einen "Mittelweg“ zwischen "Neoliberalismus" und „linker Sozialpolitik“ propagieren (Jenson 2010, S. 70). Als Hauptgegner des Sozialinvestitionsstaats können sozial-konservative Wähler und Wählerinnen und Parteien gelten, die Anstoß am progressiven Familienbild des Sozialinvestitionsstaats-Modells nehmen. Nicht zuletzt scheinen die erstarkenden Rechtspopulisten in allen europäischen Ländern ein wichtiger Gegner der Sozialinvestitionen zu werden. Durch den Fokus auf Mittelschicht-Policies fühlen sich zudem Klientelgruppen der klassischen Sozialpolitik - teilweise zu Recht - vernachlässigt und benachteiligt.

Eine weitere Besonderheit von Sozialinvestitions-Politik ist ihre (langfristige) Zukunftsorientierung und das temporale Auseinandertreten von Kosten und Erträgen. Während der Hauptnutzen kompensierender Sozialpolitik sich im Hier und Jetzt zeigt (etwa durch höhere Arbeitslosengelder oder Renten), fällt der wesentliche Teil des Nutzens von Sozialinvestitionen laut Vertretern dieses Ansatzes erst in der $\mathrm{Zu}$ kunft an. Dennoch erzeugen einige sozialinvestive Policies auch konkreten Nutzen in 
der Gegenwart. Ein gutes Beispiel ist der Ausbau staatlicher Kinderbetreuung: Dieser hat positive, gleichzeitige Effekte auf die (Frauen-)Erwerbstätigkeit. Der erhoffte langfristige Nutzen (wie bessere Ausbildung, Vermeidung von Bildungsungleichheit, höhere Beschäftigungsfähigkeit und das Abmildern neuer sozialer Risiken) zeigt sich jedoch erst, wenn die Generation in den Arbeitsmarkt eintritt, d. h. oft zwei bis drei Dekaden später. Die materiellen Kosten dieser Politiken - auch wenn diese oft vergleichsweise gering ausfallen -, sind trotzdem sofort spürbar. Politisch sind diese Zeitinkonsistenzen eine Herausforderung (Jacobs 2008), da parteipolitische Akteure sich oft an kurzfristigen Wahlzyklen und nicht am langfristigen Nutzen von Policies orientieren. Die starke Zukunftsorientierung ist somit ein weiterer Grund, warum sich die politische Dynamik der Sozialinvestitionspolitik maßgeblich von derjenigen der kompensierenden Sozialpolitik unterscheidet. Sie ähneln in dieser Hinsicht viel mehr anderen zukunftsorientierten Politiken, beispielsweise der Umweltpolitik.

\subsection{Funktioniert Sozialinvestitionspolitik? Kritische Perspektiven}

Verfechter des Sozialinvestitionsstaats schwärmen von seinen positiven Auswirkungen auf Gesellschaft wie Wirtschaft. Doch welche Effekte haben Sozialinvestitionen wirklich? Die empirische Literatur ist weitaus kritischer. Eine Hauptkritik am Sozialinvestitionsstaat ist, dass dieser nicht - wie erhofft - vor allem den neuen sozialen Risikogruppen zugutekommt, sondern diejenigen privilegiert, die ohnehin schon besser abgesichert sind. So kritisieren beispielsweise Bea Cantillon und Wim van Lancker (2013), dass insbesondere Besserverdienende vom Ausbau staatlicher Kinderbetreuung profitieren. Peter Taylor-Gooby et al. (2015) zeigen, dass Sozialinvestitionen zwar höhere Beschäftigungsraten zur Folge haben, aber nicht zur Armutsbekämpfung beitragen. Giuliano Bonoli und Fabienne Liechti (2018) finden in einer Evaluationsstudie aktiver Arbeitsmarktprogramme, dass deren Erfolg über soziale Gruppen variiert. Die Forschung spricht hier in Anlehnung an den Vers „Wer hat, dem wird gegeben“ aus dem Matthäusevangelium von „Matthäus-Effekten“.

Darüber hinaus wächst zunehmend die Kritik am Fokus der Politik und Forschungsliteratur auf den quantitativen Ausbau von Sozialinvestitionen, wohingegen beide die Qualität der entsprechenden Institutionen und sozialen Dienstleistungen $\mathrm{zu}$ wenig berücksichtigten. So wird beispielsweise immer wieder betont, dass Matthäus-Effekte besonders dann wahrscheinlich sind, wenn Leistungen nicht universell verteilt werden (wenn z. B. nicht alle Kinder Kindertagesstätten besuchen, sondern nur ein Teil von ihnen, beispielsweise die Kinder der Besserverdienenden) (van Lancker 2014). Daraus wird oft die Forderung abgeleitet, universellen Zugang anzustreben. Allerdings wird dabei übersehen, dass diese Einrichtungen die gewünschten Effekte nur dann erreichen können, wenn die Qualität ausreichend hoch ist, wenn also beispielsweise gut ausgebildete, gut bezahltes Fachpersonal Kinder in Kleingruppen betreut (zu diesem Zielkonflikt siehe auch Bothfeld und Rouault 2015). 
Eine weitere Kritik am Sozialinvestitionsmodell ist, dass Politiker oft nur ein Lippenbekenntnis zum Sozialinvestitionsmodell ablegen und in diesem Rahmen Sozialkürzungen durchführen, indem sie etwa unter dem Etikett der ,Aktivierung workfare-Maßnahmen verschärfen, d.h. anstatt Arbeitslose mit neuen Fähigkeiten auszustatten und auf, gute Jobs' vorzubereiten, diese durch Sanktionierungen möglichst schnell in jegliche Art von Arbeit zu befördern. Außerdem wird kritisiert, dass über den Fokus auf neue soziale Risikogruppen alte gefährdete Gruppen vergessen werden (Bothfeld und Rouault 2015) und ein Ausbau des Sozialinvestitionsstaats die klassischen Arbeitsmarkt-Außenseiter in noch prekäreren Verhältnissen zurücklässt.

Verteidiger des Sozialinvestitionsstaats antworten auf diese Kritik, dass Sozialinvestitionen bisher in vielen Ländern nur teilweise und unvollständig implementiert sind und daher die gewünschten Ergebnisse noch nicht oder nur teilweise erfüllen können (Hemerijck 2017). Außerdem wird argumentiert, dass auch klassische Sozialpolitik oft - aus politischen Gründen - Mittelklasse-orientiert war (Korpi und Palme 1998) und nicht zwangsläufig bessere Umverteilungsergebnisse erreicht (van Vliet und Wang 2015). Sozialinvestitionen könnten - so das Argument der Verteidiger sogar ähnlich gute soziale Effekte bei besserer ökonomischer Bilanz erreichen (Ahn und Kim 2015). Diese Diskussion ist aber in der Forschungsliteratur bei weitem noch nicht abgeschlossen (vgl. auch die Diskussionen in Hemerijck 2017).

\section{$4 \quad$ Fazit}

Dieses Kapitel hat einen Überblick über die vergleichende Bildungspolitikforschung und die Literatur zum Sozialinvestitionsstaat gegeben. Im Unterschied zur klassischen Sozialpolitikforschung sind beide Literaturen relativ jung, gewinnen aber vor dem Hintergrund aktueller sozioökonomischer Veränderungen zunehmend an Bedeutung - sowohl in der Forschung als auch in der Politik. Es ist daher davon auszugehen, dass Bildungs- und Sozialinvestitionspolitik ein immer wichtigerer Teil der Wohlfahrtsstaatsforschung sein werden. Wir haben in diesem Kapitel das Verhältnis von Bildungs-, Sozialinvestitions- und kompensierender Sozialpolitik thematisiert und herausgearbeitet, wie sich ihre jeweiligen politics unterscheiden. Wir haben außerdem beispielhaft aufgezeigt, dass sie auf komplexe Weise mit sozioökonomischer Ungleichheit zusammenhängen. In diesem Sinne kann die aktuelle Debatte um den Sozialinvestitionsstaat durchaus als Fortsetzung früherer Diskussion zum Verhältnis von Bildungs- und Sozialpolitik verstanden werden. Allerdings bleibt auch weiterhin die Analyse der Bildungspolitik an sich ein wichtiger Schwerpunkt der zukünftigen vergleichenden Wohlfahrtsstaatenforschung. Auch wenn sich die politische Dynamik der Bildungs- und Sozialinvestitionspolitik teilweise deutlich von der Dynamik klassisch umverteilender Sozialpolitik unterscheidet, eignen sich die etablierten Theorien und Methoden der vergleichenden Sozialpolitikforschung gut, um diese Unterschiede herauszuarbeiten. Mehr noch: Die Erweiterung der klassischen Analysenperspektive 
der vergleichenden Sozialpolitikforschung auf die Bildungs- und Sozialinvestitionspolitik kann maßgeblich zur Theorienbildung beitragen, insofern als deren Anwendbarkeit (scope conditions) überprüft und deren theoretische Annahmen, Erwartungen und Voraussagen verfeinert und weiterentwickelt werden.

\section{Literaturverzeichnis}

Ahn, Sang-Hoon und Kim Soo-Wan. 2015. Social investment, social service and the economic performance of welfare states. International Journal of Social Welfare 24(2): 109119.

Allmendinger, Jutta. 1989. Educational systems and labour market outcomes. European Sociological Review 5(3): 231-50.

Ansell, Ben W. 2010. From the ballot to the blackboard: The redistributive political economy of education. Cambridge: Cambridge University Press.

Antikainen, Ari. 2006. In search of the Nordic model in education. Scandinavian Journal of Educational Research 50(3): 229-43.

Barro, Robert J. 2001. Human capital and growth. American Economic Review 91(2): 12-17.

Bengtsson, Mattias, Caroline de la Porte und Kerstin Jacobsson. 2017. Labour market policy under conditions of permanent austerity: Any sign of social investment? Social Policy and Administration 51(2): 367-388.

Boix, Carles. 1998. Political parties, growth and equality: Conservative and social democratic economic strategies in the world economy. Cambridge: Cambridge University Press.

Bonoli, Giuliano. 2010. The political economy of active labor-market policy. Politics and Society $38(4): 435-457$.

Bonoli, Giuliano. 2013. The origins of active social policy: Labour market and childcare policies in a comparative perspective. Oxford, New York: Oxford University Press.

Bonoli, Giuliano und Fabienne Liechti. 2018. Good intentions and Matthew effects: Access biases in participation in active labour market policies. Journal of European Public Policy 25(6): 894-911.

Bothfeld, Silke und Sophie Rouault. 2015. Families facing the crisis: Is social investment a sustainable social policy strategy? Social Politics 22(1): 60-85.

Bouget, Denis, Hugh Frazer, Eric Marlier, Sebastiano Sabato und Bart Vanhercke. 2015. Social investment in Europe: A study on national policies. Brussels: European Commission.

Bradley, David, Evelyne Huber, Stephanie Moller, Francois Nielsen und John D. Stephens. 2003. Distribution and redistribution in postindustrial democracies. World Politics 55: 193-228.

Breen, Richard. 2005. Explaining cross-national variation in youth unemployment. European Sociological Review 21(2): 125-34. 
Busemeyer, Marius R. 2015. Skills and inequality: The political economy of education and training reforms in Western welfare states. Cambridge, New York: Cambridge University Press.

Busemeyer, Marius R. und Christine Trampusch. 2012. The political economy of collective skill formation. Oxford, New York: Oxford University Press.

Busemeyer, Marius R. und Christine Trampusch. 2011. Review article: comparative political science and the study of education. British Journal of Political Science 41(2): 413-43.

Busemeyer, Marius R. und Rita Nikolai. 2010. Education. In The Oxford handbook of the welfare state, Hrsg. Francis G. Castles, Stephan Leibfried, Jane Lewis, Herbert Obinger und Christopher Pierson, 494-508. Oxford: Oxford University Press.

Cantillon, Bea und Wim Van Lancker. 2013. Three shortcoming of the social investment perspective. Social Policy and Society 12 (4): 553-564.

Cusack, Thomas, Torben Iversen und Philipp Rehm. 2006. Risks at work: The demand and supply sides of government redistribution. Oxford Review of Economic Policy 22(3): 365-89.

Esping-Andersen, Gøsta. 1985. Politics against markets: The social democratic road to power. Princeton, New Jersey: Princeton University Press.

Esping-Andersen, Gøsta. 1990. The three worlds of welfare capitalism. Cambridge: Polity Press.

Esping-Andersen, Gøsta. 2002. Why we need a new welfare state. Oxford: Oxford University Press.

Estévez-Abe, Margarita, Torben Iversen und David Soskice. 2001. Social protection and the formation of skills: A reinterpretation of the welfare state. In Varieties of capitalism: The institutional foundations of comparative advantage, Hrsg. Peter A. Hall und David Soskice, 145-83. Oxford, New York: Oxford University Press.

EU Commission. 2013. Towards social investment for growth and cohesion. Communication from the Commission to the European Parliament, the Council, the European Economic and Social Committee and the Committee of the Regions. Brussels, 20.2.2013: EU Commission.

Garritzmann, Julian L. 2016. The political economy of higher education finance. The politics of tuition fees and subsidies in OECD countries, 1945-2015. Basingstoke: Palgrave Macmillan.

Garritzmann, Julian L., Marius R. Busemeyer und Erik Neimanns. 2018. Public demand for social investment: New supporting coalitions for welfare state reform in Western Europe? Journal of European Public Policy 25(6): 844-861.

Garritzmann, Julian L., Silja Häusermann, Bruno Palier und Christine Zollinger. 2017. WoPSI - The world politics of social investment: An international research project to explain variance in social investment agendas and social investment reforms across countries and world regions. LIEPP Working Paper 64.

Giddens, Anthony. 1998. The third way: The revival of social democracy. Cambridge: Polity Press. 
Gift, Thomas und Erik Wibbels. 2014. Reading, writing, and the regrettable status of education research in comparative politics. Annual Reviews of Political Science 17: 291-312.

Hall, Peter A. und David Soskice. 2001. An introduction to varieties of capitalism. In Varieties of capitalism: The institutional foundations of comparative advantage, Hrsg. Peter A. Hall und David Soskice, 1-68. Oxford, New York: Oxford University Press.

Häusermann, Silja. 2012. The politics of old and new social policies. In The politics of the new welfare state, Hrsg. G. Bonoli und D. Natali, 111-32. Oxford, New York: Oxford University Press.

Häusermann, Silja. 2018. The multidimensional politics of social investment in conservative welfare regimes: Family policy reform between social transfers and social investment. Journal of European Public Policy 25(6): 862-877.

Häusermann, Silja, Georg Picot und Dominik Geering. 2013. Review article: Rethinking party politics and the welfare state - Recent advances in the literature. British Journal of Political Science 43(1): 221-40.

Heidenheimer, Arnold J. 1981. Education and social security entitlements in Europe and America. In The development of welfare states in Europe and America, Hrsg. Peter Flora und Arnold J. Heidenheimer, 269-304. New Brunswick, London: Transaction Books.

Hemerijck, Anton. 2013. Changing welfare states. Oxford: Oxford University Press.

Hemerijck, Anton (Hrsg.). 2017. The uses of social investment. Oxford: Oxford University Press.

Hibbs, Douglas A. 1977. Political parties and macroeconomic policy. American Political Science Review 71(4): 1467-87.

Holliday, Ian. 2000. Productivist welfare capitalism: Social policy in East Asia. Political Studies 48: 706-623.

Iversen, Torben und David Soskice. 2001. An asset theory of social policy preferences. American Political Science Review 95(4): 875-93.

Iversen, Torben und John D. Stephens. 2008. Partisan politics, the welfare state, and three worlds of human capital formation. Comparative Political Studies 41(4-5): 60o-37.

Iversen, Torben und David Soskice. 2015. Politics for markets. Journal of European Social Policy 25(1): 76-93.

Jacobs, Alan. 2008. The politics of when: Redistribution, investment and policy making for the long term. British Journal of Political Science 38(2): 193-220.

Jenson, Jane. 2010. Diffusing ideas for after neoliberalism. The social investment perspective in Europe and Latin America. Global Social Policy 10(1): 59-84.

Kazepov, Yuri und Constanzo Ranci. 2017. Is every country fit for social investment? Italy as an adverse case. Journal of European Social Policy 27(1): 90-104.

King, Desmond. 1995. Actively seeking work? The politics of unemployment and welfare policy in the United States and Great Britain. Chicago: University of Chicago Press.

Korpi, Walter und Joakim Palme. 1998. The paradox of redistribution and strategies of equality: Welfare state institutions, inequality, and poverty in the Western countries. American Sociological Review 63(5): 661-87. 
Kuitto, Kati. 2016. From social security to social Investment? Compensating and social investment welfare policies in a life-course perspective. Journal of European Social Policy 26(5): 442-459.

Kvist, Jon. 2015. A framework for social investment strategies: Integrating generational, life course and gender perspectives in the EU social investment strategy. Comparative European Politics 13(1): 131-149.

León, Margarita und Emmanuele Pavolini. 2014. ,Social investment' or back to ,familism: The impact of the economic crisis on family and care policies in Italy and Spain. South European Society and Politics 19(3): 353-369.

Marshall, Thomas H. 1964. Class, citizenship, and social development. Garden City, N.Y.: Doubleday.

Morel, Nathalie, Bruno Palier und Joakim Palme. 2012. Beyond the welfare state as we knew it? In Towards a social investment welfare state? Ideas, policies and challenges, Hrsg. Nathalie Morel, Bruno Palier und Joakim Palme, 1-30. Bristol, UK, Chicago, IL: Policy Press.

Morgan, Kimberly J. 2006. Working mothers and the welfare state. Religion and the politics of work-family policies in Western Europe and the United States. Stanford: Stanford University Press.

Mosher, James S. 2015. Education state, welfare capitalism regimes, and politics. Comparative European Politics 13(2): 240-62.

Nikolai, Rita. 2012. Towards social investment? Patterns of public policy in the OECD world. In Towards a social investment welfare state? Ideas, policies and challenges, Hrsg. Nathalie Morel, Bruno Palier und Joakim Palme, 91-115. Bristol, UK, Chicago, IL: Policy Press.

Nolan, Brian. 2013. What use is, social investment'? Journal of European Social Policy 23(5): 459-468.

Powell, Walter W. und Kaisa Snellman. 2004. The knowledge economy. Annual Review of Sociology 30(1): 199-220.

Ronchi, Stefano. 2016. The social investment welfare expenditure data set (SIWE): A new methodology for measuring the progress of social investment in EU welfare state budgets. GK Soclife Working Paper Series 17.

Schmidt, Manfred G. 2007. Testing the retrenchment hypothesis: Educational spending, 1960-2002. In The disappearing state? Retrenchment realities in an age of globalisation, Hrsg. Francis G. Castles, 159-83. Cheltenham, Northampton: Edward Elgar.

Solga, Heike. 2014. Education, economic inequality and the promises of the social investment state. Socio-Economic Review 12(2): 269-97.

Streeck, Wolfgang. 2012. Skills and politics: General and specific. In The political economy of collective skill formation, Hrsg. Marius R. Busemeyer und Christine Trampusch, 31752. Oxford: Oxford University Press.

Taylor-Gooby, Peter, Julia M. Gumy und Adeline Otto. 2015. Can ,new welfare' address poverty through more and better jobs? Journal of Social Policy 44(1): 83-104. 
Thelen, Kathleen. 2004. How institutions evolve: The political economy of skills in Germany, Britain, the United States and Japan. Cambridge: Cambridge University Press.

Thelen, Kathleen. 2014. Varieties of liberalization and the new politics of social solidarity. Cambridge: Cambridge University Press.

Van Kersbergen, Kees. 1995. Social Capitalism: A Study of Christian democracy and the welfare state. London, New York: Routledge.

Van Kersbergen, Kees und Jonas Kraft. 2017. De-universalization and selective social investment in Scandinavia? In The uses of social investment, Hrsg. Anton Hemerijck, 216-226. Oxford: Oxford University Press.

Van Lancker, Wim. 2014. To whose benefit? An empirical and comparative investigation into the (un)intended consequences of family policy in the social investment state. Antwerpen: Universiteit Antwerpen.

Van Vliet, Olaf und Chen Wang. 2015. Social investment and poverty reduction: A comparative analysis across fifteen European countries. Journal of Social Policy 44(3): 611638.

Vlandas, Tim. 2013. Mixing apples with oranges? Partisanship and active labour market policies in Europe. Journal of European Social Policy 23(1):3-20.

Wilensky, Harold L. 1975. The welfare state and equality: Structural and ideological roots of public expenditures. Berkeley, Los Angeles: University of California Press.

Willemse, Nienke und Paul de Beer. 2012. Three worlds of educational welfare states? A comparative study of higher education systems across welfare states. Journal of European Social Policy 22(2): 105-17.

Wolf, Frieder und Reimut Zohlnhöfer. 2009. Investing in human capital? The determinants of private education expenditure in 26 OECD countries. Journal of European Social Policy 19(3): 230-44. 